\title{
The Cloud Management System of Campus Umbrella
}

\author{
Weihua He a,1, Xinyue Wang1, Bingrui Yang 2, Chai Song1, * \\ ${ }^{1}$ Institute of Electrical and Information Engineering, Southwest Minzu University of China, \\ Chengdu, Sichuan, 610041, P.R. China \\ 2 Kunming Railway Vocational Technical College, Kunming, Yunnan \\ aswunhwh@foxmail.com \\ *Corresponding author. Email: s.tschai@gmail.com
}

\begin{abstract}
Keyword: Sharing economy; Radio Frequency Identification; Credit system; Management system; Global System for Mobile communication; Database
\end{abstract}

\begin{abstract}
With the arrival of the sharing economy, the shared device appears rapid development.At present, sharing umbrella equipment lacks credit management mechanism (database), client-side, etc. The integrity of system structure is poor, the user experience is not good. RFID circuit design is simple, card reader identification is weak; Has not been established client, data feedback real time is low. The system aims at improving the accuracy of RFID system identification, strengthening database reading and writing and improving the real-time performance of data feedback.
\end{abstract}

\section{Introduction}

Whether it is capitalist society or socialist society, when describing the prospects of social development, sharing is one of the most fascinating chapter ${ }^{[1]}$. With the rapid development of sharing economy, the demand for shared devices is also increasing. This system in view of the market for umbrella Shared equipment to improve: using RFID identification, GSM data transmission, the cloud server records and client feedback, combining the ideas of the system engineering research, establish a set of consummate supervision mechanism to manage the umbrella by software rules.

As shown in Figure 1, the user is using an IC card in the lower machine to borrow the umbrella to return the umbrella. The system uses GPRS in GSM to feed the user relevant information to the database in the cloud server to read and write, after judging the user's legitimate rights, the user can give back the instructions to the lower computer, the user ID and the umbrella RFID number are interrelated, completed the borrow or return the umbrella operation, and the system services unit records every user behavior and forms a credit rating system. When the time limit exceeds the borrowing time, the credit rating is reduced and remind by SMS, the user can also inquire about the credit level and the borrowing time limit through the webpage. After analyzing the data of the leased point, the dispatching operation and maintenance information is given. The user who has repeatedly exceed time limit to returned umbrella or deliberately damaged the device, resulting in the lower credit rating of users who has stopped using the umbrella permissions. The system has the advantages of efficient management ability, low cost of management, strong human-computer interaction, etc., which is more stable and reliable than the original equipment. 


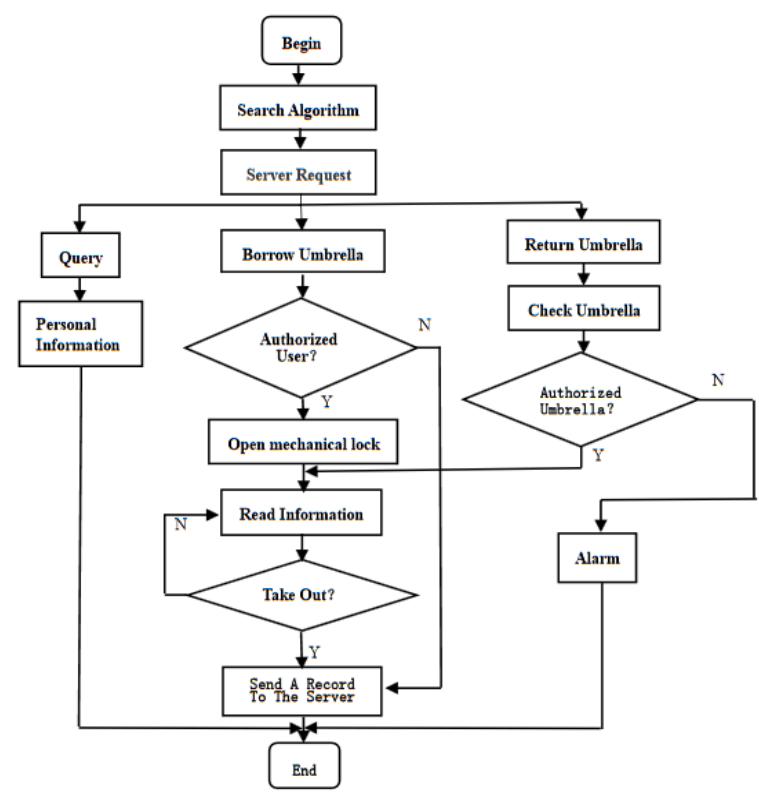

Fig. 1 System flow chart

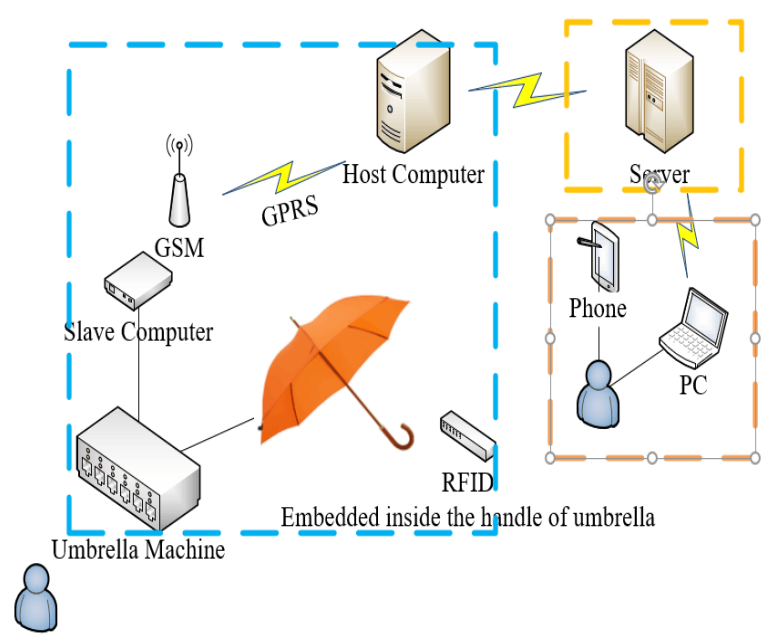

Fig. 2 The overall structure of the system

\section{Structure of a system}

The umbrella cloud management system is established under the guidance of system engineering, which is mainly composed of three subsystems ${ }^{[2]}$ : umbrella machine system, server system and user access system. The overall structure of the system is shown in Figure 1, in the blue area is the umbrella machine system; the yellow area is the server subsystem; the red area is the user access subsystem.

Using RFID identification, GSM communication, host computer and lower computer communication, database and other technologies, to achieve stable and fast mutual communication among subsystems.

\subsection{Umbrella machine system}

The mechanical framework of the umbrella system is the horizontal design, and the RFID technology is used to verify the identification of the umbrellas and users. Using GSM GPRS to realize the fast communication between the server and the upper computer STM32; Use serial communication to control mechanical locks. The frame of the system is shown in Fig2.

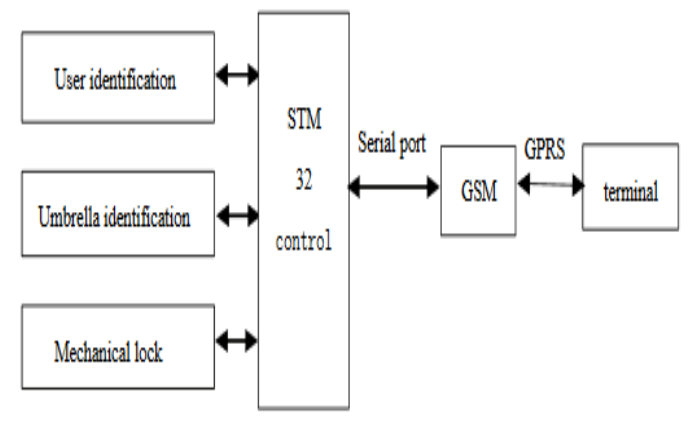

Fig.3 The frame diagram of the umbrella system

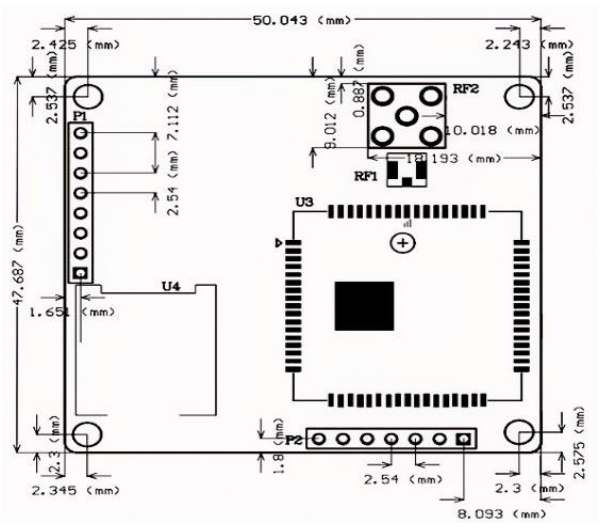

Fig.4 SIM800 dimension

\subsubsection{RFID recognition module}

In order to ensure the reflectivity of the target, the RF system generally uses high frequency 
transmission ${ }^{[2]}$. According to the communication principle ${ }^{[3][4]}$ and law of electromagnetic wave scattering ${ }^{[6]}$, assuming that the radiation power of the card reader is $P_{T X}$, the receiving power density is $\rho$, and the reflection distance is 1 . The relationship between them is as follows:

$$
l^{2}=k \frac{P_{T X} \cdot S}{16 \pi^{2} \rho}
$$

Specially, $\mathrm{S}$ is the reflection cross section area.

This system designed a contactless Read/Writer with an internal MCU, the frequency of it is 13.56 $\mathrm{Hz}$, and integrated the ISO14443A protocol, $2 \mathrm{I} / \mathrm{O}$ input and output. When the DC voltage is $3.3 \mathrm{~V}$, the current is $43 \mathrm{~mA}$, and the Baud Rate of Serial Communication is set to $9600 \mathrm{bit} / \mathrm{s}$, the reader / writer distance is about $8 \mathrm{~cm}$. The distance of reading and writing is further than existing equipment in the market.

In this system, the micro RFID tags was inserted in the he handles of the umbrella, you can monitor the card number and other information through the compassion umbrella mounted on the compassion umbrella put about $6 \mathrm{~cm}$ read, so as to prevent such behavior. The card reader was installed on the umbrella can monitor the number of umbrellas and other information, so as to prevent the behavior of changing umbrellas.

\subsubsection{Design of mechanical lock}

In order to ensure the accuracy and controllability of mechanical lock opening and closure, the system adopted Towerpro MG946R digital steering gear and mechanical slider combination as the lock control device. The design is convenient for the accurate control of the mechanical lock, and the handle can be automatically turned to the appropriate angle.

\subsubsection{Information transfer module}

In the aspect of information transmission between umbrella machine and server, taking into account the security, stability, communication speed, communication cost, and the liquidity of the machine, the information transmission between the machine and the server, the system used STM32 control GSM module to communicate with GPRS. Among them, the GSM module choose SIM800A, it has strong pass-through ability and domain name resolution ability, at the same time the volume is small (the dimension diagram is below), easy to be integrated to borrow an umbrella machine to protect.

\subsection{Server System}

The server system includes the server database, management platform and data analysis of the umbrella station. The server database contains the user IC card serial number, the RFID serial number of the love umbrella, the user's personal information, the user's borrowing / returning umbrella record, the credit value, etc. The management platform is mainly designed for the administrator, including user registration, umbrella permissions, resetting the credit value and other functions.

\subsubsection{Database server}

This system uses the open source relational database MySQL as the background data store database, and the Apache Server system environment is configured on the Linux system or Windows Server system to start the HTTP service. The back-end information processing and the overall business implementation are made up of Python script as the back-end language, using Django web as a development framework which is occupies the largest field of Web development, and it is the most popular framework. The back-end services of the entire web are configured as follows:
Apache-2.4 32bit
Python-3.4 32bit
Django-1.1 
When the user login page end registered personal account, and record its relevant information, the system will automatically match the personal registration account with the IC card serial number and enter database by register machine.

\subsubsection{Interactive server}

Interactive server has the important function of connecting the past and the future, and it is the common bridge between the lower computer and the database. The core code for receiving connection information is shown in Figure 5:

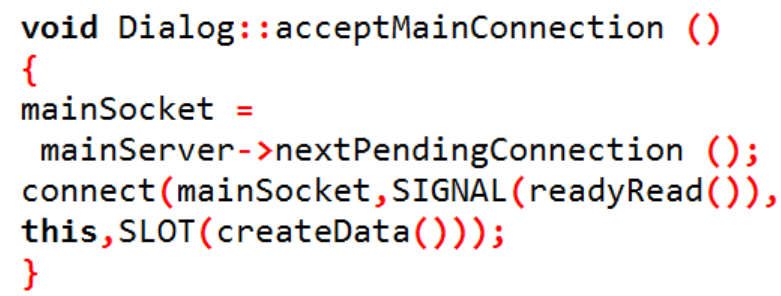

Fig. 5 core code for receiving connection information

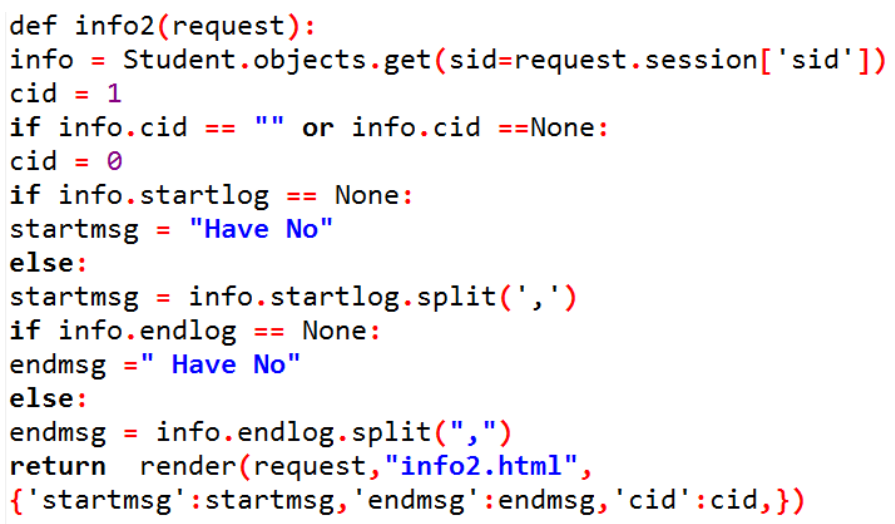

Fig.6 Part of the python back-end code

\subsection{User access system}

The user access system provides users with information such as borrowing time, credit value and borrowing/returning records. Users can login and query through the mobile terminal or the PC terminal, which avoids the cumbersome process of the existing equipment requiring users to download APP, and enhances the user friendliness. The user can at any terminal with web browsing on the visit, because web page is based on the response, the user has a good layout experience with different screen resolution.

In the user query interface, the user can query whether the umbrella is returned; when the user borrows the time beyond the preset time limit of the system, the page will be marked by the red alert. In the personal information interface, the information can be viewed and modified. Part of the python back-end code is shown in Figure 6.

\section{Conclusion}

In this paper, a sharing device is proposed, which integrates RFID, embedded, database, big data and other technologies. Compared with the original equipment, the main advantages are as follows:

1. Establish a consummate credit management mechanism(database) and the system structure is complete;

2. The interactive server is improved, and the data reading and writing between the lower computer and the database are more stable and accurate;

3. Using GPRS to do network data communication, the communication cost is low, security is high, data feedback is timely, and the equipment is mobile;

4. Independent design of RFID peripheral circuit, reading distance farther; Without the client, data feedback real-time is low.

5. Using big data analysis, providing equipment scheduling information, saving operation and maintenance costs. 


\section{Reference}

[1] Xin Meng. Shared Idea and Distributive Justice Principle [J]. Scientific Socialism, 2016(1):20-24.

[2] Xuesen Qian etc. On System Engineering [M]. Hunan Science and Technology Press, 1982.

[3] Suwen Zhang. High Frequency Electronic Circuit. Fifth Edition [M]. Higher Education Press, 2009.

[4] Changxin Fan. Communication Fundamentals. Fourth Edition [M]. National Defense Industry Press, 1995.

[5] Baosuo Shen, Chunping Hou. The Principle of Modern Communications. Second Edition[M]. National Defense Industry Press, 2006.

[6] Liqin Cao, Wei Cao. Theory of Electromagnetic Field and Electromagnetic Wave (Teaching Materials In Colleges and Universities) [M]. Science Press Publishing House Press, 2006.

[7] Nikitin P V, Rao K V S. Antennas and Propagation in UHF RFID Systems[C]// IEEE International Conference on RFID. 2008:277-288.

[8] Weimin Lang. RFID Technology Principle and Application [M]. China Machine Press, 2006.

[9] Wang H J, Su J. The Design and Research of Infrared Sensor Monitoring System Based on STM32[C]// Fourth International Conference on Computational and Information Sciences. IEEE Computer Society, 2012:876-879.

[10] Lina Qu. Design of Remote Data Acquisition and Control System for Embedded GPRS Network Based on STM32 [J]. Coal Technology,2013, 32(8):198-198.

[11] Chengtao Fan, Peifeng Gu, Jiajun Xu, et al. Multi-

networking Extensible Monitoring Module Based on STM32 (scanning tunneling microscop e)/GPRS (general packet radio service)/WIFI (wireless fidelity): CN, CN $103440756 \mathrm{~A}[\mathrm{P}]$. 2013.

[12]Wei Han, Weihong Ren, Peixin Yang, et al. Internet Application Based on GPRS Comm unication Control Technology [J]. Industrial c,2016(6):00276-00278.

[13]Jue Zhu. Design of WEB Business System Based on Linux Platform Apache, PHP, MyS QL Database [J]. China Collective Economy, 2011(21):186-187.

[14] Tang Liangrui, Cai Anni, Sun Jingao. Web Page Design From Cognitive Psychology an d Human Computer Interface, [J]. graphics, 2000 (2): 31-37. 